\section{Questión}

Periodismo / Comunicación ISSN 1669-6581
- Av. $44 \mathrm{~N}^{\circ} 676,1^{\circ}$ piso

CP 1900 - La Plata - Argentina

www.perio.unlp.edu.ar/question

El trabajo de los medios de comunicación universitarios en la pandemia. La experiencia de Radio UNDAV y UNDAV TV

Lucía Casajús y Noelia Giorgi

DOI: https://doi.org/10.24215/16696581e309

\title{
El trabajo de los medios de comunicación universitarios en la
} pandemia.

\section{La experiencia de Radio UNDAV y UNDAV TV}

The work of the university media on the pandemic. The experience of UNDAV Radio and UNDAV TV

Lucía Casajús / casajus@undav.edu.ar https://orcid.org/0000-0002-4477-4580

Doctora en Comunicación y Magíster en Periodismo Digital y Multimedia (Universidad Jaume I, España). Lic. en Periodismo y Comunicación Social (UNLP).

Responsable de articulación institucional y académica en la Dirección de Medios de la Universidad Nacional de Avellaneda. En su trayectoria profesional se ha desempeñado en medios de comunicación universitarios en Argentina y España. Sus líneas de investigación se han centrado en la radio, la radio universitaria y su desarrollo en la web 2.0. Ha publicado numerosos artículos científicos en congresos y publicaciones nacionales e internacionales; y forma parte de comités evaluadores de distintas revistas científicas de Argentina y América Latina. Es autora del libro sobre divulgación científica en la radio universitaria Lo dijo la radio. Entonces habrá que investigar y ha participado en los

libros Las radios universitarias, más allá de la radio: las TIC como recursos de interacción radiofónica y La radio universitaria como servicio público para una ciudadanía democrática, entre otros. Asimismo, ha sido docente de grado y posgrado en la Universidad Nacional de La Plata y en la Lic. en Periodismo de la Universidad Nacional de

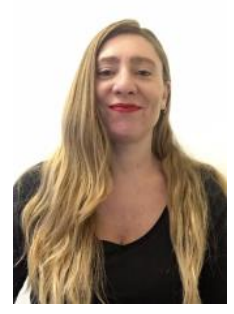


Profesora universitaria (UNLP) y maestranda en Comunicación y Creación Cultural por la Fundación Walter Benjamin. Responsable de programación de Radio UNDAV y producción general en la Dirección de Medios de la Universidad Nacional de Avellaneda, donde también es responsable de la articulación de contenidos en redes universitarias y realizadora de coproducciones junto a las emisoras Concepto Radial del Instituto Tecnológico de Monterrey (México), Onda Campus de la Universidad de Extremadura (España) y las emisoras que integran la red de Radio Internacional Universitaria (RIU). En su trayectoria laboral se ha desempañado como productora ejecutiva en programas de Radio Del Plata, Belgrano y Provincia. Ha publicado distintos artículos científicos sobre el trabajo de Radio UNDAV en congresos y publicaciones nacionales e internacionales. Es autora del libro sobre divulgación científica en la radio universitaria Lo dijo la radio. Entonces habrá que investigar y ha participado en los libros Pensar las radios: reflexiones desde las cátedras, talleres y otros alrededores; y Radios Universitarias en Marcha. Hacia la construcción de una contra agenda mediática, entre otros. Además es docente de grado en la Universidad Nacional de Avellaneda.

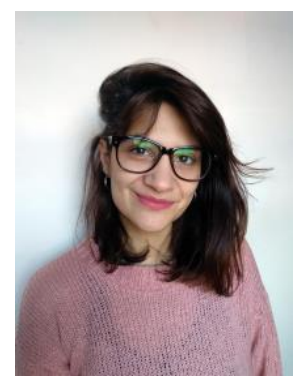

¿Cuál es el rol de los medios de comunicación universitarios en la pandemia? ¿Qué contenidos debemos garantizar? ¿Cómo se producen y emiten en el marco del Aislamiento Social Preventivo y Obligatorio? Este artículo intenta dar respuestas a esas preguntas a partir de la experiencia de trabajo de la radio y la televisión de la Universidad Nacional de Avellaneda, Radio UNDAV y UNDAV TV. Expondremos, en estas líneas, las acciones que implementamos desde la Dirección de Medios de la UNDAV, que es el área que coordina y gestiona la radio y la televisión universitaria de Avellaneda, y describiremos la implementación de distintas acciones de comunicación y articulación, y mecanismos técnicos para poder desarrollar las tareas de manera remota, mediante el teletrabajo, para realizar y emitir una programación especial para informar y dar servicio desde los medios universitarios en la emergencia por el COVID-19. 
Información y servicio: Puesta en marcha del informativo especial por COVID-19

Ante las primeras informaciones de la situación epidemiológica del COVID-19 en nuestro país, Radio UNDAV comenzó a realizar el tratamiento del tema en los programas institucionales diarios que realiza la Dirección de Medios (DDMM) en la emisora: Vivís la UNDAV y Meridiano Nacional, a partir de entrevistas a investigadores de la Universidad y a referentes nacionales del ámbito de la salud y la política. Estas entrevistas fueron compiladas en un podcast específico en la web y difundidas en las redes sociales.

La situación sanitaria se complejizaba y desde el equipo de la Dirección de Medios de la UNDAV se tomó, entonces, la decisión de comenzar a realizar un informativo especial. Así, el 18 de marzo, dos días antes de que en la Argentina se decretara el Aislamiento Social Preventivo Obligatorio, Radio UNDAV puso en marcha el Informativo Especial Coronavirus que se emite de lunes a viernes desde las 12 y hasta las 18 hs., cada una hora, y se publica también en la web y redes sociales de la emisora.

La primera tarea fue evaluar el tipo de contenidos y se estableció que debería incluir informaciones vinculadas a la emergencia con noticias del ámbito nacional, provincial, regional y local, así como comunicaciones institucionales de la Universidad Nacional de Avellaneda.

La segunda cuestión, fue ver cómo distribuir el trabajo entre el equipo, para poder realizar el informativo en forma remota y contemplando las herramientas con las que cada trabajador/a cuenta en sus casas, que no son las mismas con las que se trabaja en las instalaciones de la radio y de la señal de televisión, a nivel técnico.

A partir de estas cuestiones se decidió que participara de la producción y realización del informativo todo el equipo de la Dirección de Medios, a cargo del Loc. Mario Giorgi. Se estableció una coordinación, a cargo de la Dra. Lucía Casajús y la Prof. Noelia Giorgi; se definió que la producción y grabación de las noticias estaría a cargo del equipo de locutorxs y periodistas de la DDMM: Paula Maitía (noticias nacionales y provinciales), Axel Govednik (locales e instucionales) y Fernando Pearson (regionales). En tanto que la edición del mismo estaría a cargo de los operadores técnicos, Lautaro Federico, Matías Belli Basualdo, Diego Aguilera y Marcos Bralo, de manera rotativa y en paralelo a las tareas técnicas que realizan. Al mismo, tiempo, se dispusieron una serie de mecanismos técnicos y de articulación para poder desarrollar las tareas de manera remota y dar inicio, así, mediante el teletrabajo a la realización diaria del informativo. 
En el aspecto técnico, se programaron las dos PC's de aire para que, en caso de posibles cortes de luz, iniciaran automáticamente y se realizó la instalación de un software para acceder de manera remota a las computadoras de la emisora. Se redactó, además, un manual de procedimiento para los operadores de la radio. Paralelamente, se creó una carpeta en Google Drive compartida con todo el equipo de la Dirección de Medios, y se cargaron allí contenidos de actividades pendientes o en proceso y se realizó también, de manera preventiva, la descarga y automatización de separadores, siglas, spots, promos y artística para su posterior emisión.

En cuanto a la producción y edición del informativo, como hemos señalado anteriormente, se dispuso que el Informativo Especial Coronavirus brindara información útil y de servicio a partir de los anuncios del Gobierno Nacional, el Gobierno de la provincia de Buenos Aires, el distrito de Avellaneda y la Región, así como de las noticias institucionales de la Universidad Nacional de Avellaneda (UNDAV).

Como primera acción se pensó y redactó la artística y nombre del informativo, luego se procedió a la grabación y edición de la misma mediante los dispositivos con que cada locutor y operador del equipo cuenta en su casa.

Para la producción de noticias, se dispuso que tres locutorxs y periodistas de la Dirección de Medios, fueran los encargados de producir dos informaciones cada uno, y se les asignó el ámbito a cada unx de ellxs: Nacional y provincial; Regional; Local e institucional. También se elaboró un manual de procedimiento para su grabación y envío a la Coordinación del informativo, dentro de la cual una persona se dedica a la Producción General.

De esta manera, como señalamos anteriormente, el 18 de marzo comienza a emitirse diariamente el Informativo Especial Coronavirus, realizado íntegramente a distancia con una duración de entre 5 y 8 minutos.

El contenido de cada noticia, como mencionamos anteriormente, busca informar de manera veraz y oficial, con criterio periodístico y utilizando fuentes confiables, ya sean de los organismos nacionales o entidades encargadas de anunciar a la ciudadanía las medidas preventivas y pautas de comportamiento social, así como información institucional de la UNDAV. Siempre, teniendo en cuenta las recomendaciones realizadas por el Ente Nacional de Comunicaciones (ENACOM) que, en este sentido, expuso que "se tome conciencia de la sensibilidad que implica la comunicación. Solicitamos la colaboración de los medios para evitar 
la propagación de noticias falsas".1

Para el Informativo Especial de Radio UNDAV, pensado principalmente como un servicio a la comunidad, se dispuso también la producción de las noticias con la incorporación de los testimonios de actores involucrados en tareas de primera necesidad. Se consultan así, fuentes de las áreas de salud, servicios, sindicatos y gremios, entre otros.

Una vez recibidos los audios con las noticias, que Ixs tres periodistas y locutorxs envían a un único correo electrónico al que tienen acceso tanto la coordinación como los operadores, la productora general edita y revisa los contenidos que envía luego a la edición final. En caso de ser necesario se vuelve a grabar o se actualizan en función del surgimiento de nuevas informaciones. Cuando está terminado, la Coordinación integrada por dos personas, chequea el informativo completo, y la productora general lo carga en un Drive compartido por todos los integrantes de la DDMM, quedando el informativo listo para su emisión.

Concluida la producción, se procede a la emisión y difusión. El informativo se programa para las 12 hs. y su repetición, cada hora, hasta las 18 hs. Asimismo, hay un operador a cargo de subir el audio a la web de la emisora, en la sección de podcasts, y enviarlo por Whatsapp a la lista de distribución de Radio UNDAV. También, dentro de la coordinación, hay una persona encargada de la difusión del informativo en los canales de Facebook y Twitter de la DDMM @undavmedios, que se realiza con enlace al podcast de la web, ya que estas plataformas no permiten la subida de contenidos en audio. El informativo también puede escucharse en directo por streaming en la web de la emisoraz y es enviado a todas las emisoras universitarias argentinas a través del grupo de Whatsapp de productores de la Asociación de Radiodifusoras Universitarias Nacionales Argentinas (ARUNA), reforzando la tarea de extender la comunicación y la difusión de la información.

Educación y bienes culturales: Incorporación del Programa Seguimos Educando

El 16 de marzo, el Consejo Interuniversitario Nacional (CIN) y el Ministerio de Educación firmaron un convenio marco para la producción y difusión de contenidos pedagógicos por parte de la Asociación de Radiodifusoras Universitarias Nacionales de Argentina (ARUNA), a la que

\footnotetext{
${ }^{1}$ Comunicado ENACOM: Divulgación de información falsa 19/03/2020

https://www.enacom.gob.ar/noticias/institucional/comunicado--divulgacion-de-informacion-falsa_n2226

${ }^{2}$ http://radio.undav.edu.ar/
} 
pertenece Radio UNDAV; y la Red Nacional de Audiovisuales Universitaria (RENAU), en la que se encuentra UNDAV TV; y la Red de Editoriales Universitarias Nacionales (REUN). Todas ellas, organizaciones interuniversitarias dependientes del CIN. El convenio tuvo como objetivo el acompañamiento a las medidas del Gobierno Nacional respecto de la situación epidemiológica del coronavirus COVID-19.

De esta manera, la emisora de radio y la señal de TV oficial de la Universidad Nacional de Avellaneda, Radio UNDAV y UNDAV TV, se unieron al programa del Ministerio de Educación y RTA, SEGUIMOS EDUCANDO, en el marco de este convenio entre el CIN y el Ministerio de Educación para difundir en los medios universitarios contenidos educativos y garantizar el acceso a bienes culturales, en acompañamiento a las medidas del Gobierno Nacional.

Así, el viernes 20 de marzo, Radio UNDAV comenzó a incluir en su programación el segmento que Radio Nacional (RNA) dedica a SEGUIMOS EDUCANDO. Para ello, se realizó el relevamiento de contenidos que consistió en revisar qué programas ponía a disposición RNA, qué tipos de contenidos, qué cantidad de producciones había y cuál era la duración en cada caso. Con la selección realizada se creó una grilla de programación en emergencia y se dispuso los horarios en que debían ser programados los contenidos a incorporar. Se comenzó con la descarga y nomenclado de los contenidos y, siempre de de manera remota, se cargaron y programaron en las computadoras de aire para su emisión. Hasta la fecha se incorporaron y emitieron los siguientes programas, Leer es un placer y Contame una historia (9 hs.); Historias de Nuestra Historia (a las 11 y a las 15 hs.); Nosotros que fuimos a Malvinas y El eslabón perdido (13hs.); Hay alguien ahí (17 hs.); y Mamá Rock (19 hs.).

Por su parte, UNDAV TV, incorpora la difusión del segmento que la TV Pública dedica al programa, que se está emitiendo como contenido destacado en la web de la señal universitaria y en el canal de YouTube, en el que se ha creado una lista de reproducción específica del programa, actualizada diariamente, que cuenta actualmente con más de 200 producciones audiovisuales. UNDAV TV trabaja con dos personas. Una de ellas, es la encargada de agregar diariamente los contenidos en el canal de YouTube, a partir de los subidos por la TV Pública, actualizando así el contenido destacado de la web. La otra persona se encarga de la difusión de los contenidos en las redes sociales Facebook, Twitter e Instagram, que dan cuenta de la participación de UNDAV TV y Radio UNDAV en SEGUIMOS EDUCANDO. 
Campañas de concientización: \#En Casa

Desde la Dirección de Medios de la UNDAV concebimos como un eje central la participación y la puesta en valor de la palabra de la comunidad universitaria a través de la radio y la televisión. Por esta cuestión, en el marco del Aislamiento Social Preventivo y Obligatorio, se decidió poner en marcha desde Radio UNDAV y UNDAV TV campañas de concientización a través de las voces de la comunidad universitaria que forma parte de la programación de nuestros medios universitarios.

Así, el mismo día en que salió el Decreto del Gobierno Nacional, desde la emisora universitaria se convocó a Ixs participantes de los distintos programas que conforman la grilla de Radio UNDAV a enviar mensajes de de audio de concientización bajo la consigna "Yo me quedo en casa porque...", expresando allí lo que desearan.

Tras la extensión de la cuarentena, UNDAV TV convocó a Ixs estudiantes de la UNDAV que realizaron el programa informativo 7 Puentes en sus cuatro temporadas (2016-2019), a enviar sus mensajes de concientización a través de un video grabado con el celular bajo la consigna "Estoy estudiando y participando desde casa porque..." invitándolxs a transmitir su experiencia. Estas acciones dieron como resultado las campañas: Radio UNDAV \#YoMeQuedoEnCasa https://youtu.be/IWjozDhrtlY y 7 Puentes \#EnCasa https://youtu.be/Ap_C8UKM-b4, que fueron emitidas en los medios universitarios y difundidas, también en las redes sociales Facebook, Twitter e Instagram @undavmmedios

Programación y contenidos especiales

La Dirección de Medios también evaluó cómo adaptar las programaciones de la radio y la televisión en el nuevo contexto, teniendo en cuenta las acciones mencionadas anteriormente pero garantizando la difusión de aquellas producciones de la comunidad universitaria que fueran enviadas por quienes integran las grillas.

Así, en Radio UNDAV, se continúan recibiendo, editando y programando contenidos con diferentes temáticas que envían los programas para incorporar en la programación. Se conformaron, también, listas musicales y se incorporaron spots realizados desde la Asociación de Radiodifusoras de Universidades Nacionales Argentinas (ARUNA) sobre recomendaciones, medidas preventivas y consejos de los organismos de salud y del Ente Nacional de Comunicaciones (ENACOM), realizados en el marco de la pandemia con información útil para 
usuarios y consumidores. Diariamente se descargan y actualizan contenidos, y se programa y automatiza de forma remota para su rotación en el aire y su emisión por streaming. Asimismo, se realizan transmisiones especiales tales como la solicitada por el Departamento de Humanidades y Artes de la UNDAV, del ciclo de charlas "Conversaciones Orilleras" del que participan destacadas personalidades de distintas disciplinas, que tiene como propósito reflexionar acerca de la escena contemporánea mundial como consecuencia del COVID-19, y que se realiza los jueves a las 15 hs., en directo. También, se realizan corresponsalías para las emisoras asociadas a ARUNA con información sobre las actividades especiales, modalidades de cursada e información útil para estudiantes y trabajadores de la UNDAV, y noticias locales del partido de Avellaneda.

En UNDAV TV, también se continúan emitiendo ciclos que están realizando producciones audiovisuales en cuarentena, tales como el ciclo de conciertos de músicos/as independientes en la UNDAV, CUMI y el ciclo de música y arte La Chimenea, ambos realizados en conjunto entre la Secretaría de Extensión y el Departamento de Humanidades y Artes de la UNDAV, que son subidos semanalmente a la web y al canal de YouTube. También se continúan cargando contenidos especiales enviados a la pantalla tales como el video Las Universidades del Conurbano en la Pandemia, realizado por la UNAJ, la UNDAV, la UNQ, la UNPAZ, la UNM, la UNAHUR y la UNO.

Asimismo, desde la Dirección de Medios, se produjeron para ambos medios contenidos especiales en conmemoración del Día Nacional por la Memoria, la Verdad y la Justicia, el 24 de marzo; y del Día del Veterano y los Caídos en la Guerra de Malvinas, el 2 de abril.

Cabe mencionar, además, que se están preparando también contenidos especiales para el octavo aniversario de Radio UNDAV y el cuarto aniversario de UNDAV TV, que celebramos el 7 de mayo, aún en cuarentena y para lo cual estamos produciendo spots y videos especiales para radio, televisión y redes sociales.

Finalmente, desde la Dirección de Medios, continuamos durante la pandemia analizando, reflexionando y difundiendo el trabajo de los medios universitarios de la UNDAV a través de la elaboración de posteos en redes sobre publicaciones que hemos realizado tales como libros de la Colección Radio Escrita, realizada en conjunto con UNDAV Ediciones; participamos en virtualmente el Congreso Internacional Comunicación y Pensamiento de la Universidad de Sevilla con una exposición sobre la experiencia de los medios de comunicación de la UNDAV; 
elaboramos nuestras propias gacetillas de prensa sobre las actividades de gestión, los contenidos y las acciones de Radio UNDAV y UNDAV TV en la pandemia, y también artículos para el Periódico Con Información de la Universidad, elaborado por la Dirección de Prensa de la UNDAV, entre otras acciones de comunicación que podemos mencionar.

\section{A modo de reflexión}

Continuar con el trabajo de los medios universitarios en la pandemia, y en cuarentena, es una tarea que ha requerido esfuerzos extras y gran dedicación por parte de cada unx de Ixs trabajadores de la Dirección de Medios. Diariamente nos enfrentamos a dificultades para el desarrollo del trabajo, tanto técnicas por falta de herramientas en el ámbito doméstico, cortes de luz o del servicio de Internet; como con diferentes realidades humanas de Ixs recursos humanos que hacemos la radio y la televisión universitaria; con distintas situaciones familiares a las cuales debemos adaptar el trabajo para poder gestionar, coordinar y planificar la tarea que realizamos diariamente. A pesar de estas cuestiones, intentamos establecer horarios fijos y protocolos de trabajo, y mantener a todxs los compañerxs informados de las acciones 0 actividades. Esto requiere un tiempo extra, un compromiso y una adaptación que exceden los convenios colectivos de trabajo y que demuestran el compromiso de los trabajadorxs en esta situación de emergencia, aún en situaciones de incertidumbre y ansiedad que muchas veces convierten las actividades diarias en una tarea difícil, a la que se dedica incluso más tiempo e intensidad que en las condiciones laborales habituales.

Por otra parte, se evidencia en estos momentos la importancia de la convergencia digital. Las redes sociales, internet y los dispositivos móviles, muestran hoy su importancia y potencial como herramienta para continuar con la comunicación y la difusión de los contenidos de la radio y la televisión. En tiempos donde los medios, y sobre todos los medios universitarios, son necesarios por informar desde una comunicación inclusiva, democrática y alternativa, integrando las voces de la comunidad universitaria, actores políticos y del territorio y extenderla a la sociedad en general.

Así, Radio UNDAV y UNDAV TV siguen en el aire, a través de internet y las redes sociales con contenidos especiales y programaciones en emergencia. Seguimos informando, comunicando, difundiendo bienes culturales y garantizando el derecho a la educación, incluyendo las voces de estudiantes, docentes, investigadorxs y trabajadorxs de la universidad y actores de la 
comunidad.

Aún en la pandemia, continuamos gestionando los medios de comunicación universitarios en su doble vertiente, no solo como herramientas de comunicación, sino también como espacios que conjugan un fuerte compromiso con la educación y el aprendizaje, una función de servicio público con responsabilidad y compromiso social. Que garantizan la apertura a la participación en ellos de toda la comunidad universitaria, que visibilizan temas y actores tradicionalmente invisibilizados por los medios hegemónicos, permitiendo un tratamiento diferente y en profundidad, no solo de las actividades universitarias sino también de los temas coyunturales de actualidad informativa.

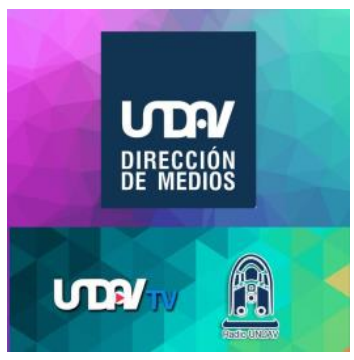

Notas 